\title{
Transrectal ultrasound (TRUS) guided prostate biopsy: Three different types of local anesthesia
}

\author{
Giuseppina Anastasi, Enrica Subba, Rosa Pappalardo, Luciano Macchione, Gioacchino Ricotta, \\ Graziella Muscarà, Francesco Lembo, Carlo Magno \\ Unit of Urology, Department of Human Pathology, University of Messina, Messina, Italy.
}

\begin{abstract}
Summary Transrectal Ultrasound (TRUS) guided prostate biopsy is regarded as the gold standard for prostate cancer diagnosis. The majority of patients perceive TRUS-guided prostate biopsy as a physically and psychologically traumatic experience. We aimed to compare in this paper the efficacy of three different anesthesia techniques to control the pain during the procedure.

Materials and methods: 150 patients who underwent transrectal ultrasound (TRUS) guided prostate biopsy were randomly divided into three groups. Group A included 50 patients who received one hour before the procedure a mixture of $2.5 \%$ lidocaine and $2.5 \%$ prilocaine, Group B: 50 patients who received intrarectal local anesthetic administration (lidocaine $5 \mathrm{ml} \mathrm{10 \% )}$ and lidocaine local spray $15 \%$ and Group C included 50 patients who received periprostatic block anesthesia (lidocaine $10 \mathrm{ml} \mathrm{10 \% ).} \mathrm{Visual} \mathrm{analogue} \mathrm{scale} \mathrm{(VAS)} \mathrm{of}$ patients in different groups was evaluated at the end of the biopsy and 30 minutes after the procedure.

Results: The VAS of patients in Group A was $1.32 \pm 0.65$ (VAS I) and $2.47 \pm 0.80$ (VAS II). In group B the VAS of patients was $1.09 \pm 0.47$ (VAS I) and $1.65 \pm 0.61$ (VAS II). In group $C$ the VAS of patients was $2.63 \pm 0.78$ (VAS I) and $1.70 \pm 0.85$ (VAS II). There was no statistically significant difference in term of VAS I between group $A$ and $B$.

A statistically significant difference was determined in terms of VAS II between group A and B. There was no statistically significant difference in term of VAS between group B and C. Conclusions: The most effective of the three methods for pain control we used was intrarectal local anesthetic administration and lidocaine local spray $15 \%$ that enables an ideal patient comfort.
\end{abstract}

KEY WORDS: Biopsy; Local anesthesia; Pain control; Prostate.

Submitted 15 November 2016; Accepted 15 December 2016

\section{INTRODUCTION}

Prostate cancer is the second leading cause of cancer death behind only lung cancer with more than 180.000 new cases/year and 25.000 deaths per year. Transrectal Ultrasound (TRUS) guided prostate biopsy is regarded as the gold standard for prostate cancer diagnosis as demonstrated by recent studies. The majority of patients perceive TRUS-guided prostate biopsy as a physically and psychologically traumatic experience (1). The International Association for the Study of Pain has offered the following definition of pain: "Pain is an unpleasant sensory and emotional experience associated with actual or potential tissue damage, or described in terms of such damage" (2). Two factors are usually responsible for pain during transrectal prostate biopsy: anal pain due to ultrasound probe, that causes pressure and stretching of muscle fibers, and pain at insertion of the needle through the prostate (3). All of the pain-rating scales are reliable and valid. The well-known visual analogue scale (VAS) and numeric rating scale (NRS) for assessment of pain intensity agree well and are equally sensitive in assessing acute pain after surgery, and they are both superior to a fourpoint verbal categorical rating scale (VRS).

Early studies conducted on sextant biopsy without anesthesia showed that $65 \%$ to $90 \%$ of patients reported discomfort (1) and 19\% would not agree to undergo it again without some form of anesthesia. Various kinds of local anesthesia have been used before TRUS-guided prostate biopsy, but there is no agreement about the most effective one. TRUS-guided biopsy is commonly performed with intrarectal local anesthetic (IRLA), but one study has shown that $>50 \%$ of patients reported moderate-tointolerable pain even with intrarectal lidocaine application before the procedure (4). The most commonly used anesthetic is lidocaine either in gel suspension or as an injectable preparation (periprostatic nerve block - PPNB) (5) although there is no strong evidence to recommend the different types of anesthetics that may be used. Some authors doesn't use any types of anesthetics during transrectal prostate biopsy. Aim of our study was to compare the efficacy of three types of anesthesia methods for a painless transrectal ultrasound (TRUS) guided prostate biopsy.

\section{Materials AND MEthods}

150 patients who underwent transrectal ultrasound (TRUS) guided prostate biopsy (12 cores) were randomly divided into three groups of 50. Group A included 50 patients who receive one hour before mixture of $2.5 \%$ lidocaine and $2.5 \%$ prilocaine, Group B included 50 patients who received intrarectal local anesthetic administration (lidocaine $5 \mathrm{ml} \mathrm{10 \% )} \mathrm{and} \mathrm{lidocaine} \mathrm{local} \mathrm{spray}$ 15\% and Group C included 50 patients who received periprostatic block anesthesia (lidocaine $10 \mathrm{ml}$ 10\%). After transrectal ultrasound examination, assessing the 
prostatic diameter, the volume of the whole prostate, the transition zone, capsular and seminal vesicle characteristics, a sampling was carried out with a 18Gauge Tru-Cut needle powered by an automatic spring-loaded biopsy disposable gun. Visual analog scale (VAS) of patients in groups was evaluated at the end of the biopsy (VAS I) and $30 \mathrm{~min}$ utes after the procedure (VAS II). The pain level was scored between 1 and 10 . In VAS scale, number 0 represented absence of pain and number 10 the maximum pain perceived in life.

\section{Results}

In Group A, Group B, and Group C, the mean age of patients included into the study was $61.4 \pm 7.9$ (47-82), $59.7 \pm 6.3(49-72)$, and $63.4 \pm 7.5(48-77)$ years, respectively. The mean age of patients in all groups was $61.5 \pm$ 7.4 (47-82) years. No statistically significant difference was observed in terms of age distribution between groups $(P=0.16)$.

The mean serum total PSA (TPSA) level of patients in Group A, Group B, and Group C was $11.2 \pm 25.9$ (4.9052) $\mathrm{ng} / \mathrm{mL}, 8.9 \pm 5.3(4.3-43.2) \mathrm{ng} / \mathrm{mL}$, and $7.6 \pm 4.1$ (5.2-36.8) ng/mL, respectively. No statistically significant difference was also observed in terms of serum TPSA level distribution between groups $(P=0.658)$.

Mean FPSA/TPSA ratio in Group A, Group B, and Group $C$ was $17 \pm 12(10-50), 18 \pm 8$ (5-33), and $20 \pm 10$ (3$55)$, respectively. No statistically significant difference was observed in terms of FPSA/TPSA ratios between groups $(P=0.598)$. The mean serum free PSA (FPSA) level of patients in Group A, Group B, and Group C was $4.53 \pm 8.10(0.8-5.0) \mathrm{ng} / \mathrm{mL}, 2.40 \pm 2(0.50-8.57)$ $\mathrm{ng} / \mathrm{mL}$, and $3.10 \pm 2.14(0.30-7.6) \mathrm{ng} / \mathrm{mL}$, respectively. No statistically significant difference was observed in terms of serum FPSA level distribution between groups $(\mathrm{P}=0.714)$.

The mean TRUS prostate volume of those in Group A was $42 \pm 12.5(30-65) \mathrm{ml}$, those in Group B was $48 \pm$ $17.3(32-69) \mathrm{mL}$, and those in Group $C$ was $45.6 \pm 15.7$ (30-63) $\mathrm{mL}$. Total TRUS prostate volume was $42 \pm 36.7$ (30-69) mL. No statistically significant difference was determined in terms of TRUS prostate volume between groups $(P=0.681)$ (Table 1$)$.

The VAS of patients in Group A was $1.32 \pm 0.65$ (VAS I) and $2.47 \pm 0.80$ (VAS II). In group B the VAS of patients was $1.09 \pm 0.47$ (VAS I) and $1.65 \pm 0.61$ (VAS II). In group $C$ the VAS of patients was $2.63 \pm 0.78$ (VAS I) and $1.70 \pm 0.85$ (VAS II). There was no statistically significant difference in term of VAS I between groups A and B. There was a statistically significant difference in term of VAS I between group C and groups A and B. There was a statistically significant difference in term of VAS II between groups A and groups B and C. There was no statistically significant difference in term of VAS II between groups B and C.

The VAS of patients in Group A was $1.32 \pm 0.65$ (VAS I) and $2.47 \pm 0.80$ (VAS II). In group B the VAS of patients was $1.09 \pm 0.47$ (VAS I) and $1.65 \pm 0.61$ (VAS II). In group $C$ the VAS of patients was $2.63 \pm 0.78$ (VAS I) and $1.70 \pm 0.85$ (VAS II) (Table 2). There was no statistically significant difference in term of VAS I between groups A and B. There was a statistically significant difference in term of VAS I between group C and groups A and B. There was a statistically significant difference in term of VAS II between groups A and groups B and C. There was no statistically significant difference in term of VAS II between groups B and C.

The cost of anesthesia was 12.90 for patient in group A; 0.25 for patient in group B; 8.50 for patient in group C (anesthesia and Chiba needle). In group A 55\% of patients in group A would agree to undergo another prostate biopsy; $65 \%$ in group B and 60\% in group C.

Table 2.

Results for mean pain scores.

\begin{tabular}{|lll}
\hline & VAS I & VAS II \\
\hline Group A & $1.32 \pm 0.65$ & $2.47 \pm 0.80$ \\
\hline Group B & $1.09 \pm 0.47$ & $1.65 \pm 0.61$ \\
\hline Group C & $2.63 \pm 0.78$ & $1.70 \pm 0.85$ \\
\hline
\end{tabular}

\section{Discussion}

It is now well accepted that TRUS-guided prostate biopsy causes significant pain and discomfort in patients and that some form of pain relief should be administered. However, given the two-way origin of pain; that is, probe-related anorectal discomfort and prostate capsule punctures, neither of the methods alone provides sufficient pain control throughout the whole process. Since first introduced by Obek et al., who showed a significantly decreased level of pain with local lidocaine gel application before PPNB, various kinds of local analgesics (local anesthetics, myorelaxants, NSAIDs, etc.) have been introduced to supplement PPNB. As the current standard technique recommended, periprostatic nerve block involved direct anesthetic infiltration of the neurovascular bundle within the Denonvilliers' fascia, which innervates the prostate (6). Meta-analytic studies have shown its efficacy compared with intrarectal local analgesia or placebo (7).

As suggested by Giannarini et al. (8), the variability of perceived discomfort might be related to differences in 
anorectal compliance. They suggested the possibility of omitting anesthesia in those patients with high anorectal compliance. Ultrasound probe geometry may influence pain perception during the prostate biopsy procedure and may be determinant in selecting patients in which some form of analgesia is needed (9). However, PPNB grants little effect in alleviating the pain associated with ultrasound probe manipulation, which was found to be a major source of discomfort during the procedure (10). Wang et al. evaluated the analgesic efficacy and safety of different combined modalities of IRLA + PPNB versus PPNB alone, showing the better analgesic efficacy of combined anesthesia with respect to PPNB alone (11). In the study performed by Raber et al. comparing intrarectal gel and placebo before biopsy, they reported that there is a statistically significant reduction in the group where anesthesia is applied with lidocaine gel during insertion of probe into rectum and taking needle biopsies compared with placebo group and that the rates of complication were similar (12). Lidocaine was the most used local anesthetic. Results of studies using lidocaine gel for IRLA showed a significantly reduced VAS score of proberelated pain; however, anesthetic infiltration and needle biopsy pain were not significantly reduced. This could be partly attributed to the short-acting nature of lidocaine. A eutectic mixture of $2.5 \%$ lidocaine and $2.5 \%$ prilocaine has recently been evaluated in several studies, and was found to be superior to typical anesthetic agents with intrarectal local administration, probably for its much longer anesthesia duration $(2-5 \mathrm{~h})$ and deeper tissue infiltration (13).

In our experience combined intrarectal local anesthetic administration (lidocaine $5 \mathrm{ml} \mathrm{10 \% )} \mathrm{and} \mathrm{lidocaine} \mathrm{local}$ spray $15 \%$ reduced the pain. According to cost evaluation this method appears less expensive.

\section{Conclusion}

There are various forms of analgesia performed by many authors in the literature in order to remove pain and discomfort of TRUS-guided prostate biopsy. We determined that the most effective method for pain control among the three methods tested in our study was intrarectal local anesthetic administration + lidocaine local spray 15 $\%$ and that it enables an ideal patient comfort.

\section{REFERENCES}

1. Nazir B Pain during transrectal ultrasound-guided prostate biopsy and the role of periprostatic nerve block: what radiologists should know Korean J Radiol. 2014; 15:543-553.

2. Merskey H, Bogduk N. Classification of chronic pain, IASP Task Force on Taxonomy. IASP Press; Seattle: 1994.

3. Maccagnano C, Scattoni V, Roscigno M, et al. Anaesthesia in transrectal prostate biopsy: which is the most effective technique. Urol Int. 2011; 87:1-13.

4. Nash PA, Bruce JE, Indudhara R, Shinohara K. Transrectal ultrasound guided prostatic nerve blockade eases systematic needle biopsy of the prostate. J Urol. 1996; 155:607-609.

5. El-Hakim A, Mouss S. CUA guidelines on prostate biopsy methodology Can Urol Assoc J. 2010; 4:89-94.

6. Obek C, Ozkan B, Tunc B, et al. Comparison of 3 different methods of anesthesia before transrectal prostate biopsy: a prospective randomized trial. J Urol. 2004; 172:502-5.

7. Hergan L, Kashefi C, Parsons JK. Local anesthetic reduces pain associated with transrectal ultrasound-guided prostate biopsy: a meta-analysis. Urology. 2007; 69:520-5.

8. Giannarini G, Autorino R, Valent F, et al. Combination of perianal-intrarectal lidocaine-prilocaine cream and periprostatic nerveblock for pain control during transrectal ultrasound guided prostate biopsy: a randomized, controlled trial. J Urol. 2009; 181:585-91.

9. Fabiani A, Tallè M, Mammana G, et al. May ultrasound probe size influence pain perception of needle piercing during transrectal prostate biopsy? A prospective evaluation. Arch Ital Urol Androl. 2016; 88:223-227.

10. McCabe JE, Hanchanale VS, Philip J, Javle PM. A randomized controlledtrial of topical glyceryl trinitrate before transrectal ultrasonography-guidedbiopsy of the prostate. BJU Int. 2007; 100:536-9.

11. Wang J, Wang L, Du Y, et al. Addition of intrarectal local analgesia toperiprostatic nerve block improves pain control for transrectal ultrasonography-guided prostate biopsy: a systematic review and meta-analysis. Int J Urol. 2015.

12. Raber M, Scattoni V, Roscigno M, et al. Perianal and intrarectal anaesthesia for transrectal biopsy of the prostate: A prospective randomized study comparing lidocaine-prilocaine cream and placebo. BJU Int. 2005; 96:1264-7.

13. Cormio L, Lorusso F, Selvaggio O, et al. Noninfiltrative anesthesia for transrectal prostate biopsy: a randomized prospective study comparing lidocaine-prilocaine cream and lidocaine-ketorolac gel. Urol Oncol. 2013; 31:68-73.

\section{Correspondence}

Giuseppina Anastasi, MD

Enrica Subba, MD

Rosa Pappalardo, MD

Luciano Macchione, MD

Gioacchino Ricotta, MD

Graziella Muscarà, MD

Francesco Lembo, MD

Carlo Magno, MD

Unit of Urology, Department of Human Pathology, University of Messina, Messina, Italy 